\title{
Información subléxica, argumental y combinatoria de los verbos saber y conocer
}

Palabras clave: Teoría del lexicón generativo, combinación léxica, combinatoria de los verbos saber y conocer, enseñanza de español como lengua extranjera

\section{Introducción}

El error de combinación léxica es muy frecuente en la enseñanza de español como lengua extranjera. A menudo tanto a alumnos como a profesores nos resulta difícil encontrar una explicación clara y concisa que justifique por qué en algunos casos la combinación de unas palabras con otras es imposible. Me refiero a ejemplos como *saber la nieve por conocer la nieve, o *saber un bar por conocer un bar, habituales en la clase de español como lengua extranjera (a partir de ahora, ELE) incluso en niveles avanzados.

Los casos fallidos de combinación léxica, entre los que se encuentra la confusión entre los usos de los verbos saber y conocer, se producen debido a un error en la interpretación semántica de estos verbos y en su uso en contexto.

En este trabajo se va a presentar una explicación que nos permita proponer reglas que posibiliten la combinación de palabras y más específicamente la combinación de los verbos saber y conocer. En concreto, algunos fundamentos de la Teoría del Lexicón Generativo (TLG a partir de ahora) pueden explicar por qué se producen combinaciones erróneas que suponen una dificultad en la enseñanza-aprendizaje del léxico. La TLG puede contribuir a explicar la lógica del error de combinación léxica de los verbos saber y conocer en el proceso de aprendizaje de lenguas, analizar las causas de este error y tal vez 
proporcionar herramientas que permitan a los profesores dar respuestas útiles a los aprendientes de ELE sobre el funcionamiento combinatorio de estos verbos y que ofrezca soluciones para evitar errores de combinación léxica.

\section{La teoría del lexicón generativo}

La Teoría del Lexicón Generativo es una teoría sobre la organización del léxico. Ha sido explicada por James Pustejovsky (1995) y revisada por Pustejovsky y Jezek (2008). Esta teoría del léxico explica por qué el lenguaje es creativo, esto es, por qué aunque el número de palabras es limitado, si utilizamos estas en distintos contextos, somos capaces de generar un número no limitado de significados y sentidos. Es decir, permite comprender la polisemia, la ambigüedad y las extensiones de significado de las palabras. Postula que las palabras codifican en su interior una información mínima estructurada en niveles. Cada uno de estos niveles especifica distinto tipo de información subléxica de la palabra; esta información junto con una serie de mecanismos de generación de significados es la que autoriza y permite la combinación de unas palabras con otras y la que ocasiona que se generen nuevos sentidos para las palabras en los distintos contextos en los que se insertan.

Para la TLG el significado de las palabras se construye a través de la interacción entre su información interna (estructura subléxica codificada en forma de rasgos semánticos) y el contexto. Es decir, el contexto influye en la construcción del significado de las palabras, pero solo si la información subléxica de estas lo permite, esto es, si las palabras contienen en su estructura interna una información sobre la que el contexto puede operar. En suma, para la TLG el significado mínimo de las palabras es lo que permite o no su combinación con otras pero también la combinación con otras palabras es lo que permite la creación de nuevos significados en determinados contextos, debido a la operación de ciertos mecanismos.

El sustantivo paquete y el verbo dormirse aportan dos ejemplos de polisemia. El nombre paquete puede interpretarse como un contenido (Se fumó todo el paquete) o como un objeto contenedor (Abrió el paquete de tabaco). La interpretación de paquete como 'contenido' depende del verbo fumar, y la interpretación de paquete como 'contenedor' es provocada por el verbo abrir. Estas interpretaciones son posibles porque en la información subléxica de paquete se codifica el rasgo semántico [овJETo], este rasgo puede especificarse al combinarse con determinadas palabras como [OBJETO DE CONTENIDO] 
o como [objeto físico contenedor]. De la misma forma, dormirse puede ser una actividad durativa (Dormirse durante doce horas), y un hecho puntual (Dormirse a las 23.00 en punto). Estos sentidos los aportan los sintagmas doce horas y a las 23.00 en punto.

La TLG postula, pues, que los distintos sentidos que tienen las palabras se hacen efectivos, se concretan, cuando entran en contexto con otras palabras, cuyos rasgos semánticos especifican una de las posibles interpretaciones de las palabras.

La TLG propone que las palabras cuentan con niveles de estructuración o codificación léxica: el nivel de estructura argumental, el nivel de estructura eventiva y el nivel de estructura de qualia. En ellos se codifican rasgos léxicos que permiten dotar de sentido a las combinaciones léxicas.

La estructura argumental especifica el número, tipo, clase semántica y realización sintáctica de los argumentos que selecciona una unidad léxica que sea predicativa. Así, el verbo comprar exige como argumento paciente un [овJETo] - comprar un libro- y como argumento agente un [INDIVIDUO] -alguien compra-; en cambio, el verbo celebrar requiere, además de un argumento agente [INDIvidUo], un argumento [EVENTO] -celebrar un concierto-.

El nivel de estructura eventiva codifica información de tipo subléxico relativa al evento: en él se especifica el tipo de evento que expresa un predicado. Pustejovsky propone tres tipos básicos de eventos: estados, procesos y transiciones. Así, desde el punto de vista eventivo, el verbo estudiar es un proceso, con duración, no delimitado, sin final, constituido por o divisible en subenventos idénticos. Beberse una cerveza, llegar o nacer son transiciones, esto es, procesos que dan lugar a un nuevo estado.

El nivel de estructura de qualia ofrece información esencial sobre los objetos y eventos aludidos por las palabras y sus relaciones. Este nivel codifica, como indican Pustejovsky y Jezek (2016) y De Miguel (2009), información sobre las características fundamentales de la entidad (objeto o evento) a la que se refiere la palabra, información del tipo «cómo llega a existir», «cuál es su constitución interna», «para qué sirve» o «en qué se diferencia formalmente el objeto aludido de otros objetos en un dominio más extenso». Lo que la estructura de qualia pretende lograr es identificar la información interna de significado lingüístico que poseen las palabras y que permite a estas cambiar su significado dependiendo del contexto en el que se inserten. Esta información semántica interna se codifica en cuatro informaciones fundamentales sobre el objeto 
referido o el evento denotado por una palabra y son: el quale formal, el quale constitutivo, el quale télico y el quale agentivo ${ }^{1}$.

Se pueden ilustrar los cuatro rasgos esenciales de la estructura de qualia con la unidad léxica tesis: 1) El quale formal de tesis nos indica que es un 'objeto de contenido'; esto es, indica la categoría a la que pertenece la entidad denotada por una palabra. 2) El quale constitutivo nos indica que contiene información, narrativa, exposición, etc. (mejora tu tesis con argumentos más sólidos; esta tesis contiene argumentos sólidos y descripciones exhaustivas sobre los temas tratados). Es decir, el quale constitutivo codifica información sobre la constitución o el interior del objeto, sus partes componentes, su material y su peso. 3) El quale télico de tesis indica que el propósito o función de una tesis es [DEFENDER]. El quale télico, pues, informa sobre el propósito o actividad para la que está pensado o hecho un objeto. 4) Por último, el quale agentivo de una tesis se define, entre otros, con el rasgo semántico [DEFENSA], ya que el quale agentivo recoge la información sobre los factores implicados en el origen o producción de un objeto.

Pustejovsky y Jezek (2016: 34) advierten, además, de que el quale agentivo es fundamental para distinguir dos tipos de entidades: las naturales y las no naturales o artefactos. Las primeras ocurren de forma natural, sin una intencionalidad; sin embargo, las entidades no naturales o artefactos son creados a través de actividades intencionales. Por ejemplo, la finalidad del corazón es 'bombear sangre', pero no es esta un fin intencional. Sin embargo, las entidades no naturales o artefactos sí poseen información agentiva. Así, un texto llega a existir a través de la escritura y una mesa a través de su construcción. La escritura y la construcción son los quales agentivos de texto y mesa respectivamente. Estos tipos de palabras se refieren a artefactos o entidades creadas para alguna funcionalidad y, por tanto, en su información subléxica codifican datos relativos al proceso de su creación y al objetivo al que se destinan; es decir, información contenida en los qualia agentivo y télico.

Por otro lado, la TLG señala que también hay palabras de tipo complejo. Son palabras que pueden pertenecer a dos categorías o entidades y, por tanto, ser definidas de dos maneras distintas. Por ejemplo, una tesis en su quale formal,

$1 \quad$ El quale, señalan Pustejovsky y Jezek (2016), es un término que hace referencia a los conceptos que evocan las palabras. Como indica De Miguel (2009: 347) la estructura de qualia está inspirada en la teoría de los aitíai propuesta en la Metafísica de Aristóteles, que propuso unos rasgos o aitíai de partes de la realidad que nos permiten interpretar el mundo. Pustejovsky se inspira en ellos para codificar lingüísticamente la información subléxica de las palabras. 
como acabamos de indicar, es un [OBJETo de CONTENIDo] o 'información' (es una tesis muy sesuda; be leído tu tesis; los argumentos de tu tesis no son sólidos). Sin embargo, también se puede definir con el rasgo [oвjeTo físico Libro] (la encuadernación de la tesis es en piel; la tesis es muy grande y no me cabe en la estantería). Las palabras de tipo complejo son siempre polisémicas, como le sucede a tesis, que es un objeto de contenido y también un objeto físico, esto es, [CONTENido] • [овJETo FÍsico LIBRo]. Este tipo de palabras contienen más de un sentido en su quale formal lo que posibilita que en determinados contextos se proyecten distintos significados o distintas interpretaciones. Así, el enunciado Me gusta mucho tu tesis es ambiguo y polisémico en su interpretación, ya que esta palabra se puede referir al formato o al contenido. La ambigüiedad se produce porque el verbo gustar puede predicar tanto de contenidos como de objetos físicos. Pero también sucede que en otros contextos solo puede seleccionarse una de las interpretaciones. Por ejemplo, en el enunciado Es una tesis un poco difícil de entender /sesuda, en el que la combinación de tesis con difícil de entender/sesuda activa o proyecta la interpretación de 'contenido'.

A veces ocurre también, postula la TLG, que una palabra del tipo simple pasa a convertirse en una de tipo complejo al combinarse con determinados predicados. Esto es lo que provoca que las palabras de tipo simple puedan resultar polisémicas al combinarse. Por ejemplo, palabras del tipo simple, como mano, pasan a convertirse en una del tipo complejo combinado con el verbo leer, (se lee información contenida en un objeto contenedor). En este caso, el verbo leer coacciona a mano -[овјEтo FÍsico]- para que sea una palabra del tipo complejo y pase a significar 'contenido' o 'información', esto es, para que sea [OBjETo físico] • [INFORMAción] en el enunciado te voy a leer la mano.

\section{Análisis subléxico del sustantivo nieve y de los verbos saber y conocer}

Uno de los ejemplos de error de combinación léxica clásico en la enseñanzaaprendizaje de ELE consiste en confundir, como se ha mencionado, los significados de saber y conocer y, por tanto, en cometer errores a la hora de seleccionar los argumentos que requiere cada uno de estos verbos. Una muestra de este tipo de error es el siguiente, cometido por una ugandesa estudiante de español, en un nivel intermedio:

Era fin de semana, además era invierno. Salimos muy temprano a estación de tren. Cogimos primer tren. Yo estaba muy 
contenta puesto que no sabía la nieve. Pero yo estaba imaginando que era como una piedra blanca.

El error consiste en utilizar la combinación *No saber la nieve por No conocer la nieve. Esto es, hay una confusión entre lo que se puede saber y lo que se puede conocer.

A continuación se presenta un análisis de la información subléxica y eventiva de las de los verbos saber y conocer y de sus argumentos, análisis inserto en el modelo léxico de la TLG para que, a la vista de las distintas informaciones subléxicas, podamos establecer los rasgos que concuerdan y explicar en esos términos el error de selección léxica que implica la expresión *saber la nieve.

Antes de realizar dicho análisis conviene aclarar también la propuesta de estructura de qualia del argumento implicado en la combinación errónea, esto es, del sustantivo nieve. El quale formal de nieve codifica que es una palabra del tipo complejo, es decir, posee dos roles semánticos: [oBJETo físico] • [EVENTo]. Es un 'objeto físico natural' y también es un 'evento natural de creación resultado de un proceso no agentivo'. Cuando nos referimos a nieve 'objeto físico natural', su quale constitutivo informa de que su constitución es agua helada (en copos o cristales blancos) y su quale agentivo codifica que llega a existir a través del evento de la congelación del agua desprendida de las nubes. Sin embargo, si nos referimos a nieve 'evento', su quale constitutivo informa de que es un [PROCESo], [-AGENTIVo], una [TRANSICIÓN] (actividad durativa) y [+ LOGRo] (estado resultado. Objeto físico natural), y el quale agentivo codifica que llega a existir a través de su desarrollo, esto es, a través de la caída del agua mediante la gravedad.

\subsection{Rasgos subléxicos mínimos y estructura argumental y eventiva de conocer}

El tipo de evento que expresan los predicados conocer y saber es un estado, esto es, un evento simple, sin dinamismo, con duración, sin fases y sin final.

Para llegar al estado de conocer, se producen dos eventos previos que provocan ese estado: 1) el evento [CONTACTAR CON UN OBJETo ESPECífico] y/o 2) el evento [ADQUIRIR LAS CARACTERÍSTICAS PROPIAS DISTINTIVAS] de un objeto o entidad.

Por ello, pensamos que los rasgos subléxicos mínimos de conocer son: 1) [HABER CONTACTADO CON] Y 2) [HABER ADQUIRIDO EL CONTENIDO CARACTERÍSTICO 
DISTINTIVo], esto es, tener el contenido característico y distintivo, constitutivo de un objeto o entidad.

Conocer predica de las entidades u objetos y de su información característica, es decir, de las propiedades de estos. Por ello selecciona como argumentos entidades que se definen con los siguientes rasgos subléxicos: [овJETo FÍsico], (contactables o experimentables), [OBJETO DE CONTENIDO CONCRETO] (específico), [+ACCESIBLE], [PROPIEDAD], [OBJETO FÍSICO RESULTADO DE UN EVENTO DE CREACIÓN] Y [ENTIDAD ABSTRACTA] (como las propiedades): Conocer $\{a$ Juan/tu casa /la universidad / el mar / la nieveł; conocer \{tus intenciones / tu carácter / la bondad y belleza de Juan / la obra de Aristóteles/ el ideario de la universidads; conocer \{la nieve /el concierto de Aranjuez de Joaquín Rodrigo\}.

Cuando conocer predica de nombres que se refieren a entidades físicas, esto es, de [OBJETO FÍsICO CONCRETO], [+ANIMADo], la concordancia léxica se produce por el mecanismo de combinación léxica que la TLG denomina selección, que es mecanismo que opera cuando el tipo semántico que requiere un predicado es satisfecho plenamente por su argumento. Así sucede con los objetos físicos concretos y animados, ya que estos objetos especifican en su estructura de qualia que tienen referentes concretos que facilitan el acceso a ellos.

Cuando conocer predica de los nombres que designan objetos de [CONTENIDO] concreto, que se materializan en un objeto físico (a través del cual su predicado conocer tiene acceso a la información característica de la que predica), se produce un mecanismo que la TLG denomina explotación. Así, la combinación conocer un relato es posible porque el predicado conocer explota la información subléxica codificada del sustantivo relato, en concreto, la información que codifican los quale télico y agentivo del nombre relato para extraer los rasgos subléxicos que exige de sus argumentos -[+OBJETo FísICo], [+ACCESIBLE], con características propias-; en concreto, el quale agentivo informa de que llega a existir a través de la escritura en un soporte de escritura. Y el quale télico codifica que este objeto artefactual de contenido está destinado a ser leído. Esto es, conocer un relato significa 'haberlo leído'.

Por otro lado, el rasgo [+PROPIEDAD] de algunas [ENTIDADEs ABSTRACTAS] facilita el acceso del predicado conocer a este tipo de entidades para seleccionarlas como argumentos (conocer el carácter/belleza de alguien). La estructura de qualia de nombres como belleza explica su concordancia léxica con conocer. E1 quale agentivo de belleza codifica que llega a existir a través de un objeto físico

2 'Haber contactado o experimentado el relato a través de la lectura'. 
natural o artefactual, esto es, se materializa y se forma mediante un agente externo, un objeto que la contiene.

Otras entidades abstractas también pueden ser seleccionadas por el verbo conocer. Así sucede, por ejemplo, con entidades abstractas que denotan [ESTADo], [SENTIMIENTO] o [SITUACIÓN], como los nombres paz, pena y tranquilidad; conocer la paz/pena/tranquilidad significa 'haber contactado o haber experimentado estos sentimientos, estados o situaciones'.

Por último, conocer también puede seleccionar nombres que designen eventos, como nieve (conocer la nieve). Como se ha indicado, el nombre nieve es una palabra del tipo complejo y, por tanto, puede asumir dos roles semánticos: [EVEnto] - [овJETo]. Esto es, puede hacer referencia a un evento y a un objeto. Prueba de ello es que es compatible tanto con modificadores que predican de objetos físicos (cuantificadores, adjetivos que se refieren a la forma, color, etc.), como con unidades léxicas que predican de eventos (empezar, terminar, tener lugar, durar...)

Cuando el nombre nieve -palabra implicada en el error de combinación que estamos analizando-, se refiere una entidad física, puede ser seleccionado por el verbo conocer, ya que este verbo predica de objetos y/o de sus características de los objetos. Por lo tanto, la combinación no conocía la nieve es correcta, en el sentido de 'no haber tenido acceso al objeto físico nieve' (no haberlo contactado ni experimentado). Conocer explota el tipo que requiere, coaccionando a su argumento nieve a asumir el rol [OBJETO FÍsICO]; esta operación es posible porque ese rol está especificado en la estructura de qualia de nieve, en concreto, en el quale formal ('objeto físico natural resultado del evento nieve').

Conocer se puede combinar, además de con los argumentos complemento indicados, con adjuntos que especifican el modo o medio de contacto con su argumento meta (y/o su información característica) y la cantidad de información que adquiere, como se ve en los siguientes ejemplos: Ya conozco a Luis, me lo acaban de presentar; conozco muy bien a Luis. Llevo 15 años trabajando con él.

El adjunto me lo acaban de presentar especifica que el contacto con el objeto ha sido directo, pero no intenso, ya que presentar posee los rasgos subléxicos [PONER EN CONTACTO POR PRIMERA VEZ] y [OFRECER INFORMACIÓN ESPECÍFICA BÁSICA SOBRE EL OBJETO PRESENTADo]. Así pues, este adjunto ayuda a focalizar la fase o primer evento necesario para llegar al estado conocer: el acceso, el contacto, el evento contacto, (y no el evento de adquirir información). 
Sin embargo, los adjuntos muy bien, llevar 15 años, y trabajar con se refieren al medio del contacto, y el grado o intensidad y por eso facilitan la focalización al segundo evento: 'adquirir información sobre el objeto'. Es decir, se focalizan los dos eventos necesarios para conocer: 'acceder' y 'adquirir información'.

Conocer puede combinarse con adjuntos como de vista, de refilón o en carne y bueso, que indican la forma en que se produce el contacto o la experiencia con el objeto y el tipo de contacto; y también con expresiones como a la perfección, a las mil maravillas, superficialmente o como la palma de la mano, que se refieren al grado, intensidad o cantidad de conocimiento: Conozco $\{$ de vista/ de refilón/en carne y bueso/intimamente/telefónicamente\} a Juan. Conozco \{a la perfección/a las mil maravillas/ superficialmente/ como la palma de la mano\} a Juan. Los adjuntos de vista, de refilón, en carne y bueso, intimamente y telefónicamente enfocan el primer evento necesario para llegar al estado conocer: 'acceder o contactar con un objeto' (enfocan la manera en que se produce el contacto y el tipo de contacto); y los adjuntos a la perfección, a las mil maravillas, superficialmente y como la palma de la mano enfocan los dos eventos: 'acceder o contactar' (a través de una manera) y 'extraer información característica sobre el objeto'.

De todos estos tipos de adjuntos mencionados - los que se refieren al contacto y a la forma de contactar e indican si el contacto es directo o no; y los que se refieren a la intensidad o el grado- los que combinan con el nombre nieve son los que se refieren al acceso o contacto directo (experimentado) con el objeto (Conozco la nieve en carne y bueso/*conozco la nieve de refilón/*conozco la nieve telefónicamente). ${ }^{3}$

\subsection{Rasgos subléxicos mínimos y estructura argumental y eventiva de saber}

A diferencia de conocer, saber posee el rasgo subléxico [TENER UNA INFORMACIÓN ESPECÍfICA]. Para llegar a este estado se produce el siguiente evento previo, el evento [ADQUIRIR UNA INFORMACIÓN ESPECÍFICA, CONCRETA] sobre un objeto o evento. Por ello, saber selecciona como complementos, palabras o expresiones que introducen o contienen y acotan [INFORMACIÓN ESPECÍFICA] sobre objetos, propiedades o eventos: Saber que \{existe la nieve / Juan fue ayer a un cumpleaños / esta novela la ba escrito Cervantes'; Saber cuándo es tu cumpleaños; Saber las causas del conflicto.

3 Pensamos que se puede «conocer la nieve en carne hueso» si se ha tenido contacto directo, se ha experimentado. 
Saber, a diferencia de conocer, no exige que su complemento sea una entidad con la que se ha establecido previamente contacto. Esto significa que para llegar al estado saber, no se realiza el evento previo 'tener contacto con el objeto' (o 'haber experimentado el objeto'). En consecuencia, saber no puede seleccionar predicados que se refieren a objetos estáticos, sino que selecciona complementos o adjuntos que introducen contenido o información concreta y específica sobre los objetos y los eventos (relativas a las actividades, las propiedades, el contenido o el contorno), como se observa en los siguientes ejemplos: Sé que el perro está enfermo / *Sé la enfermedad de tu perro; Sé cuál es el ideario de la escuela / *Sé el ideario de la escuela; Sé por qué no se ba celebrado el seminario / *Sé el seminario; Sé qué aficiones tienes / *Sé tus aficiones. En estos enunciados las palabras por qué, qué, que, y cuál introducen, contienen y acotan información específica sobre los objetos, las propiedades y los eventos (causa, lugar, manera...).

Saber también selecciona nombres que designan objetos que contienen contenido específico, como fecha, causa y borario: Sé la fecha del concierto; Séla causa de la celebración; Sé el horario de mis clases. El quale télico de estos nombres indica que su función es especificar una información concreta relativa a la localización temporal o a la causa de un evento, y se produce así la concordancia léxica. Conocer y saber se diferencian, pues, en que el primero predica de los objetos y/o de sus 'características propias y distintivas'; y el segundo predica de 'información específica' de/sobre las entidades y los eventos. Además, conocer selecciona como argumentos 'objetos físicos y de contenido susceptibles de ser contactados o experimentados'.

Por otro lado, saber puede activar también el rasgo subléxico [TENER HABILIDAD] o capacidad para realizar una actividad. En este caso, sus argumentos son unidades léxicas dinámicas, que denotan actividad agentiva y habilidad, como algunos eventos y como las disciplinas (matemáticas, gramática, etc.), que también denotan habilidades, ya que para su desarrollo se requieren competencias y destrezas: Sé \{bailar/baile\}; Sé \{matemáticas/química/cbino\}.

Cuando saber selecciona como complemento, actividades o nombres que denotan actividad, activa el sentido [TENER HABILIDAD PARA DESARROLLAR UNA ACTIVIDAD] (agentiva): Sé tocar el piano; Sé matemáticas; Sé gramática; Sé baile. Nombres como matemáticas o gramática designan disciplinas que poseen en su estructura subléxica los rasgos [ACTIVIDAd] (agentiva), [HABILIDAD], además de [CONTENIDO E INFORMACIÓN ESPECÍFICA]. El rasgo [CONTENIDO E INFORMACIÓN ESPECÍFICA] permite que este tipo de nombres sean seleccionados por saber. Pero 
a la vez se produce un mecanismo mediante el cual este tipo de argumentos introducen en su predicado los rasgos [HABILIDAD] y [ACTIVIDAD] (agentiva) y desencadenan el sentido de: 'tener habilidad para desarrollar una actividad'.

Asimismo, los nombres que denotan evento que requiere habilidad, como bai$l e$, pueden ser seleccionados por el verbo saber.

En suma, la activación en saber del sentido 'tener la habilidad' se produce mediante un mecanismo por el que nombres o verbos que denotan eventos dinámicos (como baile o canto y bailar o cantar) o que designan disciplinas, como matemáticas, gramática, química o baile y cante, y que poseen en su estructura de qualia los rasgos [ACTIVIDAD ESPECÍfICA, +AGENTIVA Y +DINÁMICA] y [+HABILIDAD], introducen en su predicado el sentido: 'tener información y habilidad para desarrollar una actividad'. En este caso se combina con: eventos como bailar, cantar, nadar, leer, escribir, relajarse o comportarse; objetos que denotan eventos dinámicos, como baile o canto; y disciplinas, como matemáticas, gramática, bistoria o baile y cante. Estas disciplinas codifican en su estructura subléxica el rasgo [ACTIVIDAD ESPECífICA, +AGENTIVA Y +DinÁmiCA]. Además, pensamos que codifican en su estructura interna que deben ser aprendidas y que requieren un proceso previo de aprendizaje o de desarrollo de habilidades; esto es, poseen el rasgo [PROCESO DE APRENDIZAJE] (o de práctica) previo al estado de 'tener la habilidad de'. Este último rasgo subléxico explica que el verbo saber predica de eventos y disciplinas como baile, álgebra, química, gramática, francés, ya que todos estos sustantivos poseen en su estructura léxica interna los rasgos [EVENTO DINÁMICO • ESPECÍfICO • +AGENTIVO • HABILIDAD • RESULTADO DE UN PRoceso PREvio]. Cuanto mayor sea el grado de especificidad y de necesidad de proceso previo de aprendizaje, mejor funciona la combinación de saber con estos eventos. Por eso, combinaciones como \#saber natación, resultan anómalas y, sin embargo, no lo son saber diseño gráfico o saber corte y confección. Esta propuesta explica por qué nieve es incompatible semánticamente con saber. No se puede *saber la nieve, porque nieve [овJETo físico] - [Evento] no satisface el tipo de argumento que requiere saber: [INFORMACIÓN ESPECÍFICA]. Tampoco el evento nieve contiene en su estructura subléxica el rasgo [ACTIVIDAD DINÁMICA AGENTIVA] ni el rasgo [HABILIDAD].

En conclusión, saber no selecciona eventos que no requieren habilidades para su desarrollo (como ver, oír o reunirse: *saber ver, *saber oír, *saber nevar, frente a, por ejemplo, saber escuchar). Tampoco predica de objetos o eventos que aunque denoten [ACTIVIDAD], como nieve, esta actividad es [-AGENTIVA]. Esto es, 
los rasgos [-AGENTIVo] y [-HABILIDAD] del evento nieve, son los que impiden que esta unidad léxica se combine con el predicado saber.

\section{Conclusiones}

La información subléxica de los verbos saber y conocer y su análisis -en términos de la Teoría del Lexicón Generativo-, así como su estructura argumental, nos han permitido explicar qué combinaciones de palabras son adecuadas cuando estos verbos entran en contexto con otras palabras y nos han aportado una explicación de los errores de combinación que se producen con estos verbos. Además, la definición subléxica y la estructura argumental de estos verbos puede aportar una solución cuando en el aula de ELE se producen errores de combinación en los que están implicados estos verbos.

Sabemos que la información subléxica mínima de conocer es [HABER ACCEDIDo A UN OBJETO] y que este verbo predica de objetos físicos, objetos de contenido y de entidades abstractas accesibles o contactables. Por eso puede combinarse con nombres como nieve, Juan, novela o belleza. Sin embargo, la información subléxica mínima de saber es [TENER INFORMACIÓN ESPECÍFICA], por haber accedido a la información (y no al objeto), y predica de contenido específico o información específica sobre los objetos y las actividades, por eso las combinaciones saber dónde está tu casa o saber de qué color es la nieve. Por otro lado, el verbo saber no predica ni de eventos, ni de objetos. Por eso, las combinaciones *saber tu casa y *saber el ganador son incorrectas, como también lo es *saber la nieve.

A partir de esta conclusión, en una clase de español para extranjeros se podría presentar la siguiente explicación para distinguir el significado de estos verbos y conocer su combinatoria:

\section{CONOCER:}

Definición: Haber adquirido, tener contenido característico de un objeto o de una entidad por haber contactado o experimentado el objeto o la entidad. Combinación: Sustantivos que se refieren a objetos de contenido, objetos físicos o propiedades susceptibles de ser contactados o experimentados. Ej.: Conocer la nieve, a Juan, un relato, un profesor, la nieve, la belleza.

\section{SABER 1:}

Definición: Poseer, tener información específica sobre un objeto, entidad o evento. Combinación: 1) Palabras que denotan información específica 
acotada. Sustantivos que se designan contenedores de una información específica y concreta de un evento, objeto, disciplina o contenido: fecha, causa, borario, regla, fórmula. Ej.: Saber el borario de... 2) Pronombres, adjetivos y conjunciones que introducen información específica sobre característica, lugar, causa, manera, identidad, momento de las actividades, objetos y eventos: que, qué, cuándo, por qué, cómo, quién. Ej.: saber qué es la nieve.

\section{SABER 2:}

Definición: Tener habilidad o destreza para realizar una actividad. Combinación: 1) Verbos que se refieren a eventos dinámicos, agentivos y para cuyo desarrollo se requiere habilidad: nadar, cantar, bailar. Ej.: Sé nadar. 2) Sustantivos que se refieren a disciplinas para cuyo desarrollo se requiere habilidad: matemáticas, gramática. Ej.: Sé gramática. »

\section{Bibliografía}

Bosque, I. (2001): «Sobre el concepto de colocación y sus límites», Lingüística Española Actual, vol. XXIII/I, 9-40.

Bosque, I (Dir.) (2004): REDES. Diccionario combinatorio del español contemporáneo. Madrid: SM.

De Miguel, E. (2009): «La Teoría del Lexicón Generativo». En: Elena De Miguel (ed.), Panorama de la lexicología. Barcelona: Ariel, 337-368.

De Miguel, E. (2012): «Properties and Internal Structure of the Lexicon: Applying the Generative Lexicon Model to Spanish». En: Marta Sanz, José María Igoa (eds.), Applying Language Science to Language Pedagogy: Contributions of Linguistics and Psycholinguistics to Second Language Teaching. Newcastle upon Tyne: Cambridge Scholars Publishing, 165-200.

De Miguel, E. (2015): «Los nombres psicológicos: propuestas de análisis en términos sub-léxicos». En: Rafael Marín (ed.), Los predicados psicológicos. Madrid: Visor, 185-222.

De Miguel, E., Batiukova, O. (2017): «Compositional mechanisms in a generative model of the lexicón». En: Sergi Torner Castells, Elisenda Bernal (eds.), Collocations and otber lexical combinations in spanish: Theoretical, Lexicograpbical and Applied perspectives. New York: Routledge: 92-113.

Gutiérrez Cuadrado, J. (dir.) (1997): Diccionario Salamanca de la lengua española. Barcelona: Círculo de Lectores. 
Higueras, M. (2011): «Lexical collocations and the learning of Spanish as a foreign language: state of the art and future projects». En: José Luis Cifuentes, Susana Rodríguez (coords.), Spanish Word formation and lexical creation. Amsterdam: John Benjamins publishing company, 437-464.

Higueras, M. (2017): «Pedagogical principles for the teaching of collocations in the foreign language classroom». En: Sergi Torner, Elisenda Bernal (eds.), Collocations and other lexical combinations in spanish: Theoretical, Lexicographical and Applied perspectives. New York: Routledge, 250-266.

Maldonado, C. (coord.) (1996): Clave. Diccionario de uso del español actual (DUEA). Madrid: S.M.

Pustejovsky, J. (1995). The Generative Lexicon. Cambridge: MIT Press.

Pustejovsky, J., Jezek, E. (2016): «Introducing qualia structure». En: Integrating Generative Lexicon and Lexical Semantic Resources [informe técnico, manuscrito inédito], Portorož, Slovenia. 23 de mayo de 2016. http://lrec2016. 1recconf.org/media/filer_public/2016/05/10/tutorialmaterial_pustejovsky.pdf (30/06/2017).

Pustejovsky J., Rumshisky A. (2010): «Mechanisms of Sense Extensions in Verbs». En: Gilles Maurice De Schryver (ed.), A Way with Words: Recent Advances in Lexical Theory and Analysis. A Festschrift for Patrick Hanks. Kampala: Menha Publishers, 1-22.

Pustejovsky J., Rumshisky A., Moszkowicz J., Batiukova O. (2008): GLML: A Generative Lexicon Markup Language, [manuscrito inédito]. Waltham (MA, U.S.): Brandeis University: http://www.cs.brandeis.edu/ cs112/ glml/papers/glml-1.o.pdf (9-11-2019).

Pustejovsky J., Rumshisky A., Moszkowicz J., Batiukova O. (2009): «GLML: Annotating Argument Selection and Coercion». En: Harry Bunt, Volha Petukhova, Sander Wubben (eds.), Proceedings of the 8th International Conference on Computational Semantics. Países Bajos: Universidad de Tilburg, 169-18o. 
Ana Sanz Tordesillas

IES Palas Atenea

\section{Sublexical, argumental and combinatory information of verbs saber and conocer}

Key words: Generative lexicon theory, lexical combination, verbs saber y conocer, Spanish as a second language

The verbs saber and conocer and their lexical combinations generate many problems in Spanish as a second language. The Generative Lexicon explains which combinations are wrong and which one are right and consequently can be a great help when it comes to explain the combinations with these verbs. The difference between both verbs lies in the selection of different types of arguments. Conocer selects objects and/or their own and distinctive characteristics. Saber, however, selects specific information of/about objects, entities, events and words that denote agentive activity. 
Ana Sanz Tordesillas

IES Palas Atenea

\section{Subleksikalne, vezljivostne in sopojavljalne lastnosti glagolov saber in conocer}

Ključne besede: teorija generativnega leksikona, sopojavljanje besed, glagola saber in conocer, poučevanje španščine kot tujega jezika

Glagola saber in conocer in njuno sopojavljanje $\mathrm{z}$ drugimi besedami pri pouku španščine kot tujega jezika povzročajo nemalo težav. Generativni leksikon je teorija o besedišču, po kateri besede vsebujejo subleksikalne prvine, ki jim bodisi omogočajo bodisi preprečujejo sopojavljanje z drugimi besedami. Prav zato lahko dana teorija olajša poučevanje-učenje sopojavljalnih lastnosti glagolov saber in conocer. Razlika med obema glagoloma namreč izhaja prav iz dejstva, da izbirata različne vrste argumentov: conocer izbira predmete in/ali njihove 'lastne in razlikovalne značilnosti', saber pa 'specifične informacije' predmetov, stvari in dogodkov oz. o predmetih, stvareh in dogodkih ter dejavnosti ali samostalnike, ki označujejo dejavnosti, ki predvidevajo vršilca dejanja. 